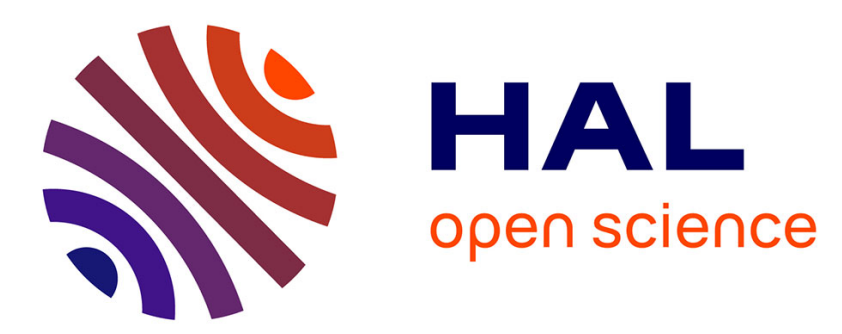

\title{
Temporalités sociales et gestion de la distance à travers les moyens de transport rapides \\ Olivier Klein
}

\section{To cite this version:}

Olivier Klein. Temporalités sociales et gestion de la distance à travers les moyens de transport rapides. MONTULET Bertrand, HUBERT Michel, JEMELIN Christophe, SCHMITZ Serge (Eds.). Mobilités et temporalités, Première partie: Transformation des temps sociaux, Facultés universitaires SaintLouis, pp. 21-33, 2005, Coll. Travaux et Recherches, $n^{\circ}$ 51. halshs-00091072

\section{HAL Id: halshs-00091072 https://shs.hal.science/halshs-00091072}

Submitted on 24 Jul 2012

HAL is a multi-disciplinary open access archive for the deposit and dissemination of scientific research documents, whether they are published or not. The documents may come from teaching and research institutions in France or abroad, or from public or private research centers.
L'archive ouverte pluridisciplinaire HAL, est destinée au dépôt et à la diffusion de documents scientifiques de niveau recherche, publiés ou non, émanant des établissements d'enseignement et de recherche français ou étrangers, des laboratoires publics ou privés. 


\section{TEMPORALITES SOCIALES ET GESTION DE LA DISTANCE A TRAVERS LES MOYENS DE TRANSPORT RAPIDES}

Olivier KLEIN, enseignant-chercheur à l'Ecole Nationale des Travaux Publics de l'Etat-Laboratoire d'Economie des Transports

rue Maurice Audin - 69518 Vaulx-en-Velin cedex

tel : (33) 0472047718 fax : (33) 0472047092

mél : olivier.klein@entpe.fr

\section{INTRODUCTION}

L’objectif de cet article est d'éclairer comment les moyens de transport à grande vitesse contemporains - on considérera surtout le TGV - répondent aujourd'hui aux structures temporelles de la société. On s’appuiera pour cela sur la distinction de différentes constructions collectives du temps, des temps sociaux.

La première partie s’attachera à décrire comment le capitalisme industriel a généré un temps industriel compatible avec la conception du travail qu’il implique. On verra que ce modèle évolue mais conserve une forte pertinence au regard de certains aspects importants des structures socio-économiques contemporaines. Pourtant, cette permanence ne semble pas en mesure de rendre compte de la totalité des évolutions actuelles. La seconde partie illustrera comment les comportements de déplacement à grande vitesse - on se limitera aux déplacements à motif professionnels - révèlent ainsi un certain nombre de ruptures fondamentales avec le modèle du temps industriel. En troisième partie, le modèle du temps fragmenté qui viendrait peu à peu se surimposer au premier, sera alors proposé. Enfin, à travers les figures de la grande vitesse comme opportunité et de la grande vitesse comme nécessité, la dernière partie présentera une lecture duale, adaptée à la double structure temporelle qui prévaudrait aujourd'hui, des modalités de gestion de la distance à travers les moyens de transport rapides. 


\section{1- $\quad$ LE TEMPS INDUSTRIEL}

\section{LE TEMPS DU CAPITALISME INDUSTRIEL}

Dans un article paru en 1979 en français, Edward THOMPSON donne une analyse détaillée de la progressive adaptation de l'appréhension du temps aux nécessités du capitalisme industriel. Il oppose le temps « orienté par la tâche » plutôt caractéristique de la société préindustrielle, au temps continu qui peu à peu va s’imposer en s’appuyant sur le développement d'une forte demande de force de travail indifférencié destinée à l’industrie.

Cette demande implique aussi que le temps soit mesurable en une unité universelle et que cette mesure soit facile : «bien sûr, comme on peut s'y attendre, c'est au moment même où la révolution industrielle exigeait une plus grande synchronisation du travail que l'usage des montres et des pendules se généralise » (THOMPSON, 1979, p. 17).

Cette évolution de la structure du temps de travail porte en elle un paradoxe fort. Elle implique en effet une adaptation « de l'ensemble des rythmes de la vie en société » (PRONOvOsT, 1996, p. 32) aux exigences de la production industrielle. Mais, simultanément, elle conduit à une rupture nette entre le temps de travail, vendu aux entrepreneurs, et le temps « libre », bien que longtemps limité aux strictes nécessités de la reproduction de la force de travail.

Pour autant, le travail ne se banalise pas. En revanche, s'appuyant sur cette dissociation entre le temps de travail et le temps « de la vie », apparaît une forte pression à la diminution du premier face au second (TONNEAU, 1998). Gerhard Bosch (1999) met en évidence combien les progrès obtenus sur ce point ont à la fois été rendus possibles et alimenté les gains de productivité réalisés durant le XXe siècle.

En résumé, le temps industriel est d'abord une marchandise, du travail qui s'échange contre un prix. Parce qu'il fait l'objet d'un marché, ce temps se structure peu à peu de manière à être continu, indifférencié, afin d'être mesuré. Il s’agit donc d'un temps exclusivement dévolu au travail dans le cadre du contrat salarial. Les autres activités sont repoussées à ses marges et les rythmes de la vie sociale s'ordonnent en conséquence, autour du travail. Valeurs centrales de la société, le travail et sa durée sont aussi au cœur du conflit d'intérêt entre ceux qui vendent leur force de travail et ceux qui l'achètent. La forte diminution du temps de travail et l'augmentation de son prix depuis un siècle ont ainsi accompagné la croissance de la productivité. 


\section{LE TEMPS INDUSTRIEL DEMEURE D’ACTUALITE}

Tel qu'il émerge des analyses historiques, le modèle du temps industriel demande bien sûr à être actualisé. En premier lieu, le travail s'est diversifié. La complexité croissante des processus de production, le développement des activités tertiaires ont été accompagnés par le développement - par acquisition d'expérience ou par formation initiale - de multiples compétences au sein de la main d'œuvre. Le marché du travail est depuis longtemps largement segmenté.

D’une «vision puritaine à une vision instrumentale du travail» (REZsOHAZY, 1986,p. 44), la représentation du travail a également largement évolué sous le coup de la croissance générale du temps libre, des revenus et du niveau de connaissance. Cette adaptation à un contexte idéologique plus individualiste marque davantage un renouvellement de la valeur sociale du travail que son affaiblissement (MORIN et CHERRE, 1999).

Cette transformation du travail se décline à travers l'évolution des modes d'organisation de la production, mais aussi à travers des exigences de souplesse, de maîtrise de ses rythmes porteuses de divergences de vue et d'intérêts (ThemMES et DE TERSSAC, 1997).

L'une des figures organisationnelles actuelles du système productif a pu être décrite, sous le terme de « taylorisme flexible » (DuRAND, 1991), comme un mode de flexibilité quantitative reposant très largement sur celle du volume de main d'œuvre, sans que les relations entre temps de travail et temps libre changent de nature. Dans ce cadre, l'accomplissement des individus est alors d'autant mieux repoussé en dehors du travail que le temps de travail est difficilement celui de la construction d'identités sociales fortes.

Ces questions prennent un relief particulier à propos du travail féminin. Même s'il n'est pas nouveau que les femmes assument une part très importante des activités humaines (SCHWEITZER, 2002), l'augmentation du nombre de femmes salariées accentue les difficultés à concilier le temps du travail et celui de la vie. Dominique MEDA (2001) insiste sur la prégnance de cette préoccupation chez les femmes. Cette tension, très largement gérée dans le cadre de la séparation du champ du travail et du champ de la vie personnelle n'est pas sans conséquence: les femmes évitent - ou sont exclues - des fonctions impliquant une forte disponibilité, elles travaillent à temps partiel, elles n'accèdent pas facilement à la formation continue (BOLTANSKI et CHIAPELLO, 1999, p. 301).

Sous réserve des contraintes de rythmes et d'horaire induite par les exigences de flexibilité de la sphère productive (TREMBLAY et VILLENEUVE, 
1997), le temps libre reste disposé autour du temps de travail. Les interférences entre les deux restent limitées aux déterminants sociaux du contenu des loisirs (GERSHUNY 1992). Néanmoins, le simple accroissement quantitatif du temps hors travail permet aujourd'hui qu'il soit le réceptacle de processus de construction d'identités collectives (YonNET, 1999). Le taylorisme flexible est donc porteur d'éléments d'évolution de la dissociation entre travail et vie sociale. Pourtant, en négociant les contraintes de manière unilatérale, du premier terme vers le second, il ne permet pas qu'elle soit remise en cause de manière fondamentale.

Le modèle du temps industriel connaît donc aujourd'hui des évolutions importantes. Il reste néanmoins cohérent et pertinent par rapport à l'ensemble des comportements économiques principalement dictés par une pression concurrentielle sur les prix (VELTZ 1993). Les stratégies visant à augmenter la vitesse de rotation des capitaux sont ainsi fondées sur les principales caractéristiques du temps industriel, continu, largement indifférencié et dépositaire d'une valeur marchande qu'exprime le taux d'intérêt. La pression sur le rendement du travail qui en résulte habituellement repose en large partie sur une représentation selon laquelle les ressources du travail ne sont pas assez spécifiques pour devenir non substituables. Le temps industriel correspond à une déclinaison flexible du taylorisme dont les sociologues du travail avouent qu'il constitue plutôt la norme du paysage économique français (LINHART, LINHART et MALAN, 1998).

\section{2- $\quad$ LES FRACTURES DE LA GRANDE VITESSE}

Malgré cette pertinence, il semble que le modèle du temps industriel ne suffise pas à comprendre la totalité des évolutions du monde contemporain. Cette partie vise à illustrer comment le TGV et les pratiques sociales qu'il supporte révèlent plusieurs points de fractures avec la représentation du temps industriel. En s’attachant aux déplacements à motif professionnel, elle envisage d'abord le développement de comportements de mobilité «pseudo ubiquistes », fondés sur la réalisation de déplacements en aller-retour sur la journée. Elle montre ensuite que ce type de comportement implique que s'estompe la frontière entre vie professionnelle et vie privée. Elle pointe enfin la systématisation de l'urgence qui s’impose comme norme sociale de fonctionnement.

Les observations menées par le Laboratoire d'Economie des Transports autour des TGV sud-est (BonNAFOUS, 1987) puis Atlantique (KLEIN et 
ClAisse, 1997) confirment la tendance au raccourcissement de la durée des voyages, les séjours d'affaires plus longs laissant la place aux allers-retours inscrits dans la journée lorsque les temps de trajets ne dépassent pas trois heures, afin de rendre disponible à destination d'une journée de travail presque intégrale.

Dans ces conditions, l’aller-retour dans la journée s’impose en tant que pratique de déplacement tout à la fois comme optimum et comme norme chez les usagers fréquents du TGV (CHEVALLIER, 1989). On peut souligner que cette dimension normative n'est pas spécifique des allers-retours dans la journée en TGV : elle concerne plus généralement les pratiques d'usage des moyens de communication les performants (CARRE, 1996 ; MEGLIN, 1996, JAUREGUIBERRY, 1996 à propos du téléphone portable).

Le relatif effacement du temps de trajet (se déplacer sans empiéter sur les activités adjacentes) évoque une «pseudo-ubiquité » qui rompt avec la continuité propre au temps industriel. Mais l'effacement demeure partiel : C'est à travers l'ordonnancement des activités, en articulant obligations professionnelles, contraintes de déplacement et vie familiale que l'on va chercher à placer dans sa journée les cinq heures de déplacement qu'impliquent un aller-retour entre Paris et Lyon (CHEVALLIER, 1989). Ces arrangements renforcent la dépendance des deux univers du travail et de la vie privée que le temps industriel avait si nettement distingué. Ils sont aussi peu accessibles aux femmes (KLEIN, 2004).

Le voyage, raccourci, peut s'envisager au dernier moment. Le TGV apporte donc une souplesse accrue dans la gestion de l'éloignement. Mais la fonction protectrice que jouait la distance ayant disparue, il devient difficile de décliner une sollicitation. Mais c’est surtout la systématisation de l'urgence que mentionnent les personnes confrontées aux outils modernes de communication (JAUREGUIBERRY, 1998 concernant le téléphone, CheVAllier, 1989 pour le TGV).

A travers ces remises en cause assez profonde de propriétés qui lui sont essentielles, on mesure ce que l'analyse de la mobilité d'affaires à grande vitesse peut révéler des fractures du temps industriel.

\section{3- LE TEMPS FRAGMENTE}

Edward THOMPSON (1979) souligne que les modèles de représentation du temps qu'il envisage ne sont jamais hégémoniques à une époque et dans une société donnée. Pour Jacques AtTALi (1982) aussi, « un Temps n’en a jamais 
éliminé un autre ; tout Temps nouveau s'est superposé à celui qui dominait avant lui » (p. 247-8). Ce passage dans la durée d'une structure temporelle à l'autre avec un large recouvrement est largement confirmé par les historiens (LEDUC, 1999, pp. 150-151) ou le sociologue Roger SuE (1995, pp. 136140). Ainsi, on peut envisager que s'affirme, à côté du temps industriel, une autre structure temporelle, encore en devenir, mais déjà distincte.

\section{LES NOUVELLES TEMPORALITES DU SYSTEME PRODUCTIF}

La pression à l'intensification de l'usage du temps est une dimension constitutive du capitalisme. Elle ne semble pas moins vive aujourd'hui qu'au $\mathrm{XIX}^{\mathrm{e}}$ siècle mais se radicaliserait. Dans la sphère économique, par exemple, la combinaison d'une concurrence hors coût, par la réactivité et l'innovation, et d'une concurrence par les coûts (Veltz, 1993, KLEIN, 2001) n'a pas seulement comme effet d'accroître la pression à réduire les délais. Elle tend aussi à structurer le temps de manière binaire où l'important n'est pas uniquement d'arriver le plus vite possible, mais surtout d'arriver « juste-àtemps » (KLEIN, 2004).

L'image d'un temps fragmenté peut être introduite à propos des rythmes quotidiens d'activité au sein même d'un contexte général de chasse aux temps morts (COHEN, 1999). DE ConincK (1995, p. 267) analyse comment l'exacerbation des exigences de synchronisation à l'intérieur d'un processus de production amène les opérateurs et leurs responsables immédiats à se heurter à un enchevêtrement de temporalités de court, moyen et long terme. La fragmentation du temps est donc une autre manière de lire la diversification des rythmes sociaux dont la régulation collective est de plus en plus difficile.

Une recomposition profonde des relations entre le temps travaillé et le temps libre apparaît également. Là où le temps industriel établit une distinction nette, d'autres rythmes contemporains semblent permettre de larges recouvrements et interdépendances (Pronovost, 1996, p. 42). Les rythmes des personnels qualifiés, structurés par une forte disponibilité et une large autonomie personnelle (BOUfFARTIGUE et BOCCHINO, 1998, p. 45) illustrent cette dilution des limites. Les formes nouvelles d'organisation du travail qui accompagnent le télétravail impliquent elles aussi lourdement les deux sphères professionnelle et domestique (STEWARD, 2000 et TIERZE et Musson 2002). 
Enfin, dans les expériences de divers pays occidentaux de réduction du temps de travail, la diminution du nombre d'heures ouvrées semble assez généralement la contrepartie d'une flexibilité horaire accrue (ToNNEAU, 1998). Que les salariés préfèrent souvent allonger leurs week-ends ou leurs congés plutôt que de réduire la durée moyenne travaillée chaque jour (ANXO et alii, 1998 ; TREMBLAY et VILLENEUVE, 1998) est souvent analysée comme le résultat d'une préoccupation liée à la protection de son temps personnel face à la fragilité des horaires normaux d'un jour de travail habituel. Elle marque aussi un renouvellement des valeurs associées au "temps libre" (DUMAZEDIER, 1988) et n'est pas indépendante d'un certain renouvellement des processus de stratification sociale (GERSHUNY, 2000, ainsi que les résultats d'enquête de Coulangeon, Menger et ROHariK, 2002). La structure duale proposée, entre temps industriel et temps fragmenté, permet d'éviter une vision trop univoque qui rattacherait la dichotomie travail/loisir à un passé fordiste et révolu (BREEDVELD, 1996, par exemple).

Une seconde remise en cause du modèle du temps industriel concerne la continuité qui lui est attachée. L'enchevêtrement des rythmes sont des images qui reviennent souvent lorsqu'il s'agit de décrire les organisations productives (ZuCCHERMAGlio et TALAMO, 2000) ou le déroulement de l'activité des individus. Dans certaines situations encore bien spécifiques, relatives en particulier aux emplois hautement qualifiés, la combinaison de différents facteurs renforce ces évolutions au point de paraître revenir au travail « orienté par la tâche » qu'Edward THOMPSON rattache aux sociétés préindustrielles. En réalité cette figure, qui constitue peut-être l'aboutissement des tendances actuelles, diffère sensiblement de celle qui prévalait dans les sociétés traditionnelles en particulier parce qu'elle se conjugue à une rationalisation systématique de l'usage du temps.

Ces ruptures concernent aussi les temps longs des carrières professionnelles. L'apprenti apprenait son métier sur le tas en même temps que les règles de conduite du milieu au contact des plus anciens. Il acquérait une identité sociale reconnue et passait l'essentiel de sa vie active dans la même usine, gravissant les échelons (souvent quelques-uns seulement) d'une hiérarchie largement stabilisée (DE CONINCK, 1994). Aujourd'hui, de restructurations en saisies d'opportunités, la carrière-type se construit souvent par séquences de quelques années, voire quelques mois. Mieux adaptée à ce rythme, la formation au coup par coup développe des compétences que la transmission d'expérience ne peut plus satisfaire. Frédéric DE CONINCK évoque à ce propos "une gestion dans le présent " (1995, p. 241). 
Enfin, la montée des incertitudes est le troisième élément de différenciation de la structure contemporaine du temps par rapport au temps du capitalisme industriel. La crise de la régulation fordiste et la pression concurrentielle avivée qui lui est consécutive jouent de ce point de vue un rôle essentiel. La recherche systématique de flexibilité devient dans ce contexte autant une réponse à cette incertitude qu'un élément de son approfondissement.

La représentation contemporaine du temps fragmenté n'est donc pas exempte de contradictions. Elle s'appuie en particulier sur un modèle organisationnel qui porte ces contradictions mais permet aussi de les gérer à travers deux tendances opposées, à l'intégration d'une part et à l'autonomie de l'autre (KLEIN, 2001). Du point de vue des structures temporelles, on peut avancer que la forte pression à l'intégration des diverses composantes d'un ensemble productif est porteuse des exigences de réactivité. En revanche, l'autonomie de chacune des composantes vise à favoriser la mobilisation de leur ressources propres, souvent développées dans la durée.

\section{4- TEMPS SOCIAUX ET MOBILITE}

Les structures temporelles de la société contemporaine sont évidemment en rapport étroit avec les comportements de mobilité que l'on peut observer en son sein. En effet, la mobilité est une pratique du temps comme de l'espace. A ce titre, elle est une partie constitutive des représentations sociales du temps qui prévalent à une époque donnée. Cette dernière partie tentera de présenter une lecture des logiques d'usage de la vitesse repérables aujourd'hui en s'appuyant sur les deux structures contemporaines du temps qui viennent d’être évoquées.

Mais avant de les distinguer, il convient de souligner que temps industriel et temps fragmenté traduisent, chacun avec ses caractéristiques, une pression croissante à l'intensification de l'usage du temps. Le développement d'un nouveau mode de transport comme le TGV, dont la première justification sociale est la vitesse, constitue alors un événement que l'on peut interpréter dans l'une ou l'autre des représentations dominantes du temps. Dans cet esprit, deux logiques d'usage de la vitesse seront proposées, la vitesse comme opportunité, qui renvoie plutôt au temps industriel, et la vitesse comme nécessité, articulée au temps fractionné. Elles prolongent les réflexions de Maurice Chevallier (1989, p.5), qui distinguait «deux types d'effetTGV » dont on verra qu'ils sont très proches des notions d'opportunité et de nécessité proposées ici : 
« - Le TGV (vitesse + fréquence) en tant que déclencheur d’une mobilité différente $[\ldots]$;

- [ou] simple aubaine (gain de temps essentiellement)»

\section{LA LOGIQUE INDUSTRIELLE DE LA VITESSE COMME OPPORTUNITE}

En première acception, le TGV est pris comme un moyen de «gagner du temps ». L'expression signifie que le temps "gagné » peut être valorisé. L'usage de la vitesse, dans cette logique, renvoie plutôt à un temps continu et échangeable, au temps industriel. Dans ce cadre temporel continu, la grande vitesse apparaît comme une opportunité propre à réduire les temps de trajet, alors considérés comme des coûts associés aux déplacements. Dans une telle logique et concernant les déplacements à motif professionnel, les coûts de déplacements peuvent être assimilés à des coûts de production. Réduire les temps de trajet revient donc à gagner en productivité. En ce sens, la vitesse comme opportunité répond en premier lieu aux exigences des activités économiques pour lesquelles la concurrence porte principalement sur les coûts. On peut donc aussi la relier au taylorisme flexible.

Cette logique de réduction des coûts explique le transfert massif de l'avion vers le train opéré à la faveur de la mise en service du TGV par les voyageurs à motif professionnel appartenant à une entreprise industrielle. Suite à l'accroissement des vitesses du chemin de fer, l'arbitrage entre les tarifs et le temps de parcours s'est trouvé déplacé suivant une logique de coût généralisé du déplacement. Pour certaines activités de services sophistiqués, cette logique se traduit plutôt par une extension des aires de marché à la faveur de la mise en service du TGV entre Paris et Lyon (BuIsson 1986) révélant une fluidité accrue des localisations d'activités

L'usage de la vitesse comme opportunité s'inscrit dans une logique de valorisation (KLEIN, 1995). Elle intègre la distance comme une contrainte de fonctionnement parmi d'autres, qui fait alors l'objet d'arbitrages en partie déterminés par les coûts de franchissement dont la vitesse est une composante. Cet arbitrage possible implique donc que l'activité qui le motive n’est pas fondamentalement dépendante des performances maximales du système de transport: il signifie que d'autres configurations sont envisageables, avec des temps de transports plus longs, qui ne remettent pourtant pas en cause l'existence, ni les principales caractéristiques de l'activité considérée. 
La vitesse comme opportunité n'est qu'une figure théorique de l'usage de la grande vitesse, tout comme le temps industriel et le taylorisme flexible ne sont que des figures théoriques des temps sociaux contemporains et des configurations organisationnelles du système productif. En effet, ces situations dans lesquelles la vitesse n'est porteuse d'aucun changement organisationnel concernant la production et la réalisation de l'activité qui motive le déplacement ne peuvent être que des fictions tant organisation, usage du temps et de l'espace sont indissociables. On peut proposer, afin de rendre compte de cette globalité, de considérer la vitesse comme opportunité comme caractéristique des situations dans lesquelles une organisation reste en mesure d'intégrer parmi ses paramètres le franchissement de la distance comme un coût.

\section{LE TEMPS FRAGMENTE DE LA VITESSE COMME NECESSITE}

La double nature d'optimum et de norme sociale de la réalisation des déplacements professionnels en aller-retour sur la journée a déjà été soulignée. Ce contexte est alors très favorable à l'incorporation, au sein même des organisations, des «facilités » de déplacement offertes par la grande vitesse.

On peut imaginer de multiples exemples d'arrangements organisationnels fondés sur cette capacité du TGV à rendre presque insensible le temps de trajet, et donc la distance (KLEIN, 2004). Ils constituent aussi pour les firmes un moyen de s'adapter à l'incertitude de leur environnement. Or l'adoption de tels schémas transforme profondément la façon dont l'éloignement peut être géré. La solidarité organique ainsi établie entre des unités géographiquement dispersées rend la grande vitesse parfaitement nécessaire pour que l'ensemble puisse fonctionner. En ce sens, la vitesse comme nécessité est caractéristique des organisations fondées sur une négation de la distance.

La vitesse comme nécessité peut alors être interprétée comme une composante de la radicalisation du processus d'intensification du temps. Elle traduit une certaine réalité selon laquelle le temps de trajet est non seulement objectivement réduit, mais où son résidu n'aurait plus d'existence car incorporé par les organisations et par les individus (à travers, pour ces derniers, des arrangements touchant à la fois les activités professionnelles et la vie personnelle). La vitesse comme nécessité renvoie alors de manière assez claire aux grands traits retenus pour décrire le temps fragmenté. 
Mais la négation de la distance qui la porte est aussi largement cohérente avec le modèle organisationnel évoqué autour des deux termes d'autonomie et d'intégration. On peut en effet considérer l'ancrage territorial (ZIMMERMAN, 1998) d'une unité de production comme une modalité de sauvegarde de sa capacité à produire des ressources spécifiques (COLLETIS et PECQueur, 1993). En ce sens, l'ancrage territorial est une marge d'autonomie. La grande vitesse est alors l'une des conditions à travers lesquelles cette autonomie peut être articulée à la pression intégrative d'une organisation couvrant plusieurs sites.

Au sein d'un cadre d'analyse rassemblant la figure du temps fragmenté et le modèle organisationnel autonomie/intégration, la vitesse comme nécessité apparaît, au côté de la vitesse comme opportunité pour compléter la grille de lecture des temps sociaux contemporains.

\section{CONCLUSION}

L'apport que tente la présente contribution est de rapprocher ces deux images de l'usage du TGV de systèmes d'analyse plus larges. Les temps sociaux industriel ou fragmenté, et plus encore le « taylorisme flexible » et le principe d' «autonomie/intégration », ont pu être définis de manière totalement indépendante de la sphère des transports. Leur mobilisation permet d'affiner l'analyse des logiques d'usage de la grande vitesse et surtout de construire une signification à une lecture en termes d'opportunité et de nécessité. En retour, l'analyse des usages sociaux de la grande vitesse permet peut-être d'envisager différemment le rôle des objets techniques de la communication dans les processus de transformations qui affectent les territoires productifs et les organisations sociales (KLEIN, 2003).

\section{BIBLIOGRAPHIE}

D. ANXO, J.-Y. BOULIN, M. LALLEMENT, G. LEFEVRE et R. SILVERA, 1998, "Recomposition du temps de travail, rythmes sociaux et modes de vie. Une comparaison France -Suède", Travail et Emploi, n 74 , pp. 5-20.

J. ATTALI, 1982, Histoires du temps, Fayard, Le livre de poche, coll. Biblio/Essais, Paris, 318 p.

L. BOLTANSKI et E. CHIAPELLO, 1999, Le nouvel esprit du capitalisme. Paris : Gallimard, coll. nrf essais, 843 p. 
A. BONNAFOUS, 1987, "The régional impact of the TGV", Transportation, Vol. 14, n 2, pp.127-137.

G. BOSCH, 1999, "Le temps de travail: tendances et nouvelles problématiques", Revue internationale du travail, vol. 138, n² 2, pp.141162.

P. BOUFFARTIGUE et M. BOCCHINO, 1998, "Travailler sans compter son temps ? Les cadres et le temps de travail", Travail et Emploi, $n^{\circ}$ 74, pp. 3750.

K. BREEDVELD, 1996, "Post-fordist leisure and work", Loisir et société, vol. 19, n 1 , pp 67-90.

M.-A. BUISSON, 1986, Effets indirects du T.G.V. et transformations du tertiaire supérieur en Rhône-Alpes, Laboratoire d'Économie des Transports, coll. Études \& Recherches, Lyon, 87 p.

D. CARRE, 1996, "Contribution à l'analyse du processus d'institutionnalisation des techniques mobiles dans le champ de la communication d'entreprise", Technologies de l'Information et Société, vol 8, $\mathrm{n}^{\circ}$ 2, pp. 189-207.

M. CHEVALLIER, avec la collaboration de Bruno CoUTURIER, 1989, $\mathrm{La}$ redistribution du temps et de l'espace des usagers fréquents $d u T G V$ à Lyon et à Valence - De l'effet TGV à l'outil TGV, Rapport de recherche pour le compte du Ministère de l'Equipement, ARCADES, Bron, 142 p.

D. COHEN, 1999, Nos temps modernes, Flammarion, coll. Essais, Paris, $161 \mathrm{p}$.

G. COLLETIS et B. PECQUEUR, 1993, "Intégration des espaces et quasiintégration des firmes: vers de nouvelles rencontres productives", Revue d’Économie Régionale et Urbaine, n³, pp. 489-508.

F. DE CONINCK, 1994, "Le temps raccourci: quand les changements prennent de vitesse le temps d'une vie", Gérer et comprendre - Annales des Mines, juin. Article repris par Problèmes économiques, $\mathrm{n}^{\circ} 2396,28$ septembre 1994, pp. 22-27.

F. DE CONINCK, 1995, Travail intégré, société éclatée, Presses Universitaires de France, coll. Le sociologue, Paris, 289 p.

P. COULANGEON, P.-M. MENGER et I. ROHARIK, 2002, 'Les loisirs des actifs: un reflet de la stratification sociale', Economie et Statistique , n ${ }^{\circ} 352-$ 353, pp. 39-55. 
J. DUMAZEDIER, 1988, Révolution culturelle du temps libre - 1968-1988, Méridiens Klincksieck, coll Sociétés, Paris, 312 p.

J.-P. DURAND, 1991, "Travail contre technologie" in Jean-Pierre DURAND et François-Xavier MERRIEN, Sortie de siècle - La France en mutation, Vigot, Paris, pp. 33-75.

J. GERSHUNY, 1992, "La répartition du temps dans les sociétés postindustrielles", Futuribles, mai-juin, pp. 215-226.

F. JAUREGUIBERRY, 1996, "De l'usage des téléphones portatifs comme expérience du dédoublement et de l'accélération du temps", Technologies de l'Information et Société, vol 8, n² 2, pp. 169-187.

F. JAUREGUIBERRY, 1998, "Télécommunication et généralisation de l’urgence", Science de la société, n 44, pp. 83-96.

O. KLEIN, 1995, " Du rôle des villes dans la constitution du réseau à grande vitesse: entre nécessité et valorisation", in Laboratoire d'Economie des Transports, Villes et TGV - actes des entretiens Jacques Cartier (Lyon, 8-10 décembre 1993), Laboratoire d'Economie des Transports, coll. Etudes \& Recherches, $n^{\circ}$ 5, Lyon, pp. 267-274.

O. KLEIN, 2001, Les horizons de la grande vitesse : le TGV, une innovation lue à travers les mutations de son époque, Thèse de Doctorat es sciences économiques, Université Lumière-Lyon 2, 310 p. Edition électronique consultée à l'adresse suivante :

http://theses.univ-lyon2.fr/bases/detail theses.php?titre=299.

O. KLEIN, 2003, 'Le travail métropolitain : un outil géographique pour révéler l'usage sélectif de la grande vitesse', L'espace géographique, $\mathrm{n}^{\circ} 2$, pp. 133-127.

O. KLEIN, 2004, "Social Perception of Time, Distancies and High Speed Transportation", Time \& Society, vol.13, n²/3, Sage publications, pp. 245263.

O. KLEIN et G. CLAISSE, avec la collaboration de Pascal PoCHET, 1997, Le TGV-Atlantique : entre récession et concurrence, Laboratoire d'Economie des Transports, coll. Etudes \& Recherches, Lyon, 163 p.

J. LEDUC, 1999, Les historien et le temps - Conception, problématiques, écritures, Le Seuil, coll. Point Histoire, Paris, 328 p.

D. LINHART, R. LINHART et A. MALAN, 1998, "Syndicats et organisation du travail : un rendez-vous manqué", Sociologie et sociétés, vol. $\mathrm{XXX}, \mathrm{n}^{\circ}$ 2, automne, 14 p. Edition électronique consultée le 28 juillet 1999 à l'adresse suivante : http://www.erudit.org/erudit/socsoc/v30n02/index.html 
D. MEDA, 2001, Le temps des femmes : pour un nouveau partage des rôles, Flammarion, coll. Essais, Paris, 199 p.

P. MEGLIN et alii, 1996, "La mobilité, entre ubiquité et omniprésence Eléménts de problématique", in Actes des séminaires Action Scientifique, "Communication mobile professionnelle", France-Télécom-CNET, n6, mai, pp. 14-43.

E. M. MORIN et B. CHERRE, 1999, "Les cadres face au sens du travail", Revue française de gestion, $\mathrm{n}^{\circ} 126$, novembre-décembre, pp. 83-93.

G. PRONOVOST, 1996, Sociologie du temps, De Boeck Université, coll. Ouvertures sociologiques, Paris, Bruxelles, 183 p.

R. REZSOHAZY, 1986, "Les mutations sociales récentes et les changements de la conception du temps", Revue Internationale des Sciences Sociales, $\mathrm{n}^{\circ}$ 107, pp. 37-52.

S. SCHWEITZER, 2002, Les femmes ont toujours travaillé : une histoire du travail des femmes au XIXe et XXe siècles, Editions Odile Jacob, coll. Histoire, Paris, 330 p.

B. STEWARD, 2000, 'Changing Time: The meaning, measurement and use of time in teleworking’, Time \& Society, vol. 09, $n^{\circ} 1$, march, pp. 57-74.

R. SUE, 1995, Temps et ordre social, Presses Universitaires de France, coll. Le sociologue, Paris, 320 p.

J. THOEMMES et G. DE TERSSAC, 1997, "La négociation du temps de travail et les composantes du référentiel temporel", Loisir et société, Vol. 20, $\mathrm{n}^{\circ} 1$, pp. 51-72.

E. P. THOMPSON, 1979, "Temps, travail et capitalisme industriel", Libre, $\mathrm{n}^{\circ} 5$, pp. 3-63.

S. TIETZE et G. MUSSON, 2002, "When "Work" meets "Home”: Temporal flexibility as lived experience', Time \& Society, vol. $11, \mathrm{n}^{\circ} 2$, september, pp. 315-334.

D. TONNEAU, 1998, "Création et temps de travail: vingt ans de perspectives", Réalités industrielles, novembre, pp. 22-26.

D.-G. TREMBLAY et D. VILLENEUVE, 1997, "Aménagement et réduction du temps de travail : réconcilier emploi, famille et vie personnelle", Loisir et société, vol. 20, $\mathrm{n}^{\circ} 1$, pp. 107-157.

D.-G. TREMBLAY et D. VILLENEUVE, 1998, "De la réduction à la polarisation des temps de travail : des enjeux de société", Loisir et société, vol. 21, n², pp. 399-416. 
P. VELTZ, 1993, "D'une géographie des coûts à une géographie de l'organisation; Quelques thèses sur l'évolution des rapports entreprises/territoires", Revue Economique, n4, juillet, pp. 671-684.

P. YONNET, 1999, Travail, loisir. Temps libre et lien social, Gallimard, coll. Bibliothèque des sciences humaines, Paris, 324 p.

J.-B. ZIMMERMAN, 1998, "Nomadisme et ancrage territorial : propositions méthodologiques pour l'analyse des relations firmes-territoires", Revue d'Économie Régionale et Urbaine, $\mathrm{n}^{\circ}$ 2, pp. 211-230.

C. ZUCCHERMAGLIO et A. TALAMO, 2000, 'The Social Construction of Work Time: negociated time and expected time', Time \& Society, vol. 09, $\mathrm{n}^{\circ} 2$, september, pp. 205-222. 